Kovacs, G. 1985, Acta Astron., 35, 37

Kovacs, G. and Buchler, J.R. 1988 ApJ, 324, 1026

Kovacs, G., Buchler, J.R., and Marom, A. 1991, A\&A, 252, L27

Kovacs, G.; Buchler, J.R., Marom, A., Iglesias, C.A., and Rogers, F.J. 1992, A\&A, 259, L46

Lee, Y-W., Demarque, P., and Zinn, R.J. 1990, ApJ, 350, 155

Petersen, J.O. 1991, A\&A, 243, 246

Petersen, J.O. 1992, A\&A, 265, 555

Sandage, A.R. 1993, AJ, 106, 703

Sandage, A.R. 1987, The Second Conference on Faint Blue Stars, eds. A.G. D. Philip, D.S.

Hayes, and J.W. Liebert (Schenectady, L. Davis Press), p.41

Simon, N.L., and Cox, A.N. 1991, ApJ, 376, 717

Strom, J., Carney, B.W., and Latham, D.W. 1992, PASP, 104, 591

\title{
CEPHEIDS: PULSATION, EVOLUTION, OPACITY
}

\author{
NORMAN R. SIMON \\ Department of Physics and Astronomy, University of Nebraska-Lincoln
}

With the advent of new radiative opacities, the gross discrepancies in period ratio between observed stars and theoretical models have been eliminated in both the "beat" and "bump" Cepheid regimes (Moskalik, et al. 1992; Kanbur \& Simon 1994). This paves the way for detailed modeling of the beat and bump stars.

We begin here by summarizing one such effort, the full description of which is given by Simon \& Kanbur (1994). In this study, the slope of the evolutionary luminosity-mass relation is taken to be $\operatorname{dog} L / \operatorname{dog} M=4.0$ (Stothers and Chin 1991) and the intercept determined by forcing models which follow this slope to also conform with the bump Cepheid resonance condition $P_{2} / P_{0}=0.50$ at $P_{0}=10 d$ (Simon \& Schmidt 1976; Buchler $=e t$ al. 1990). We then model separately each of the dozen known galactic beat Cepheids, subject to the just mentioned evolutionary constraint imposed by the 10 -day resonance. We find masses and luminosities, ranging from $M=4 \mathrm{M}_{\odot}, \log L=3.0$ at $P_{0}=3 d$ to $M=6 \mathrm{M}_{\odot}$, $\log L=3.5$ at $P_{0}=10 \mathrm{~d}$. Furthermore, a range of metallicity, $0.01<Z<0.02$, is required among the beat stars, whose period ratios, $P_{1} / P_{0}$, are predicted to decrease as the metallicity rises. This trend has been observed (Andrievsky et al. 1993). However, "standard" evolutionary tracks for $Z=0.02$ [e.g., the Geneva calculations (Schaller, et al. 1992) or the Padua calculations (Bressan, et al. 1993)] cannot produce Cepheids with parameters as given above - first because the models are somewhat underluminous at given mass, and second because the calculated blue loops do not reach the instability strip for $M=7 \mathrm{M}_{\odot}$. On the other hand, the models described by El Eid (1994) do penetrate the strip at masses as low as 4 or $5 \mathrm{M}_{\odot}$, but display a luminosity-to-mass ratio considerably too small to satisfy the 
resonance condition for bump Cepheids. Possible resolutions to these problems include a deep Fe convection zone whose effect would be to lower the period ratios (Cox 1993), and/or an opacity increase near $1 \times 10^{6} \mathrm{~K}$, which could extend the blue loops (Stothers \& Chin 1994). Each of these schemes require more research.

Finally, we point out a difficulty involving the long-period Cepheids, $P_{0}>20 d$. Because these stars do not seem to be exceedingly rare, either in the Galaxy or Magellanic clouds, they should correspond to "slow" evolutionary timescales, which are generally associated with the tips of the blue loops. However, for $M>7 \mathrm{M}_{\odot}$ (high masses are necessary to give the long periods in question, for reasonable values of $T_{e f i}$, the evolutionary tracks display loops which are too long, so that the tips occur well blueward of the instability strip. This means that for the requisite masses, passage through the instability strip is so rapid that few longperiod Cephids ought to be seen. At metallicities corresponding to those of the LMC and SMC, respectively, all of the long-period Cepheid sample in the Clouds is to be accounted for, a way will be needed to slow the evolution of high mass models through the strip.

Andrievsky, S.M., Kovtjukh, V.V. Makarenko, E.N. \& Usenko, I.A. 1993, MNRAS, 265,257 Bressan, A., Fagotto, F., Bertelli, G. \& Chiosi, C. 1993, A \& AS, 100, 647

Buchler, J.R., Moskalik, P. \& Kovacs, G. 1990, ApJ, 351, 617

Cox, A.N. 1993, in New Perspectives on Stellar Pulsations and Pulsating Variable Stars, ed. J.M. Nemec \& J.M. Matthews (Cambridge: Cambridge University Press), 261

El Eid, M.F. 1994, A\&A, 285, 915

Kanbur, S.M. \& Simon, N.R. 1994, ApJ 420, 800

Moskalik, P., Buchler, J.R. \& Marom, A. 1992, ApJ, 385, 685

Schaller, G. Schaerer, D., Meynet, G. \& Maeder, A. 1992, A\&AS, 96, 269

Simon, N.R. \& Kanbur, S.M. 1994, ApJ, in press (July 10)

Simon, N.R. \& Schmidt, E.G. 1976, ApJ, 205, 162

Stothers, R.B. \& Chin, C.-W. 1991, ApJ, 381, L67

Stothers, R.B. \& Chin, C.-W. 1994, preprint

\section{CEPHEID VARIABLES: PERIOD AND MASS DETERMINATION}

\section{PAWEL MOSKALIK}

\section{Copernicus Astronomical Center, ul. Bartycka 18, 00-716 Warszawa, Poland}

Determination of masses has been a long standing problem in the Cepheid research.

Since the early days of Cepheid modeling different methods of mass calibration have lead to conflicting results, implying serious discrepancies between the evolution and pulsation theories (see Cox 1980 for a review). In recent years this situtation has been mostly remedied, and the Baade-Wesselink masses, pulsation masses and evolutionary masses are now in good agreement with each other (e.g., Gieren 1989). However, both the bump 